\title{
Review On: Train Scheduling Using Simulation
}

\author{
Mahendra Makeshwar ${ }^{1}$, Rasika Patil ${ }^{2}$, Pooja Meshram ${ }^{3}$, Sneha Farkade ${ }^{4}$, Damini Jambhulkar ${ }^{5}$ \\ ${ }^{1}$ Assistant professor, ${ }^{2,3,4,5}$ UG Student \\ Department of Information Technology, Nagpur Institute of Technology, Nagpur, India,440026
}

\begin{abstract}
In this paper present by Train Scheduling using Simulation. This paper basically works on Scheduling and Simulation. This paper are feasible timetable generator framework for stochastic simulation modelling is developed. The main objective is feasible train timetable for all trains in the system, which includes train arrival and departure times at all visited station and calculated average train travel time. Dynamic performance of the train movement is modelled with the help of Newton's law of motion. Train movement in this work is based on four operating modes: 1) accelerating mode, 2) constant speed or cruising mode, 3) coasting mode and 4) braking mode. this concept for train movement simulator class is described with its properties and methods. Single track train scheduling problem is set of trains from opposite sides along a single track. Each train connects two given stations along the track and may have to stop for a minimum time in some of the intermediate stations. Train can overtake each other only in correspondence of an intermediate station and a minimum time interval between two consecutive departures and arrivals of train in each station is specified. Four object of array variables are used in the simulation process to keep train and station data. Two object arrays are used for the train movements in up and down directions. The stations' data are stored in the other two object arrays.
\end{abstract}

Keywords- Train scheduling; model predictive control (MPC); predictive scheduling; (PS); centralized traffic control (CTC)

\section{I- INTRODUCTION}

$\mathbf{I}_{\mathrm{n}}$ this paper we consider a train scheduling (timetabling) problem is the problem of determining a timetable for a set of trains that do not violate track capacities and satisfies some operational constraints. A general train scheduling problem in a literature considers a single track linking two major stations with a number of intermediate stations. A train simulation also called as railroad simulation is a computer base simulation of rail transport operation. They are 3D virtual reality world. Train simulation and scheduling system has a useful impact on rail crop organization of Australia. Four object of array variables are used in the simulation process to keep train and station data. Two object arrays are used for the train movements in up and down directions. The stations' data are stored in the other two object arrays. One of these arrays of station contains all the stations of the line while the other one contains only the stations with siding facilities. First come first served (FCFS) dispatching rule is used to select one train among the candidate trains, which are the trains waiting at neighbour stations of the track that want to use the same track and has finished waiting for dwell time and additional unplanned delay time. Namely the candidate trains are the trains that deserved to begin checking the conditions. If the all conditions to move are suitable for a candidate train, which arrived first to one of the neighbour station of track it will begin to trip, else the same check is made for another train arrived second. Train speed are used in railway operation and research applications, such as train performance calculation, journey time estimation, energy consumption evaluation and train scheduling, new route planning, old route upgrading. Train scheduling is one of the most interesting problems in transportation planning systems. Train scheduling problem is a single track linking two major station with a number of intermediate stations in between timetable generator simulation model. Train scheduling is closely related to machine scheduling. The train scheduling problem faced by railway infrastructure managers during real time traffic 
control. Rail driver is a desktop cab controller for train simulation software. Today railway simulation tool open track is well established railway planning software and it is used by railway suppling industries consultancy and universities in different countries. Timetable construction analyzing the robustness of timetables.

Four algorithms are developed for solving a train scheduling problem. The speed of each algorithm is given as a Big-O function of the problem size (number of train), n. Some information is also given about the quality of the solution they find, a) Rank this algorithm in term of their speed.

- Algorithm 1: $\mathrm{O}(\log \mathrm{n})$; does not guarantees anything but sometimes gives the optimal solution.

- Algorithm 2: $\mathrm{O}\left(2^{\mathrm{n}}\right)$; performance is unknown.

- Algorithm 3: $\mathrm{O}(\mathrm{n})$; guarantees finding a feasible solution.

Track failure is an event that prevents a train to occupy the impaired track for a trip. The train can use the track after it is repaired. In the simulation model, the track failures are controlled via variables. If a failure occurs in a track part, trains are prevented to use this part until it is repaired.

\section{II- LITERATURE SURVEY}

This paper as per the view of many researchers work by train scheduling using simulation including:

(a) S Yang in 2018 deals with "Energy-efficient timetable and speed profile optimization with multi-phase speed limits" focused on Energy consumption and associated costs, energyefficient timetable and speed profile optimization for metro system have attracted much attention from both academic and industry communities. This paper develops an integrating timetable.

(b) MA Murtaza, SBL Garg, in train simulation 1989, "Brake modelling in train simulation". This paper deals with brake modelling as used in train simulation study.

(c) Landex and Nielsen (2013), earlier train scheduling was done by the humen experts but now with the introduction of IT and new technological tools, it is done with the help of such technological algorithms. The system keeps a check on the synchronized railway tracks and help to run the railway with the help of algorithm. The system allows scheduling of three lines of railway, i.e. it can manage the running of three railway, at a time.

(d) According to Li etal. (2013), scheduling of trains helps to reducing the operation time as earlier most of the man power gets wasted in such processes but now it is done with ease. This helps organization to enhance the performance as the use of technology make work force to focus on their assigned duties rather than such scheduling processes. The complete process of scheduling the train is dependent on algorithm were the change of track, running of trains and related activities are managed with the help of algorithm.

\section{III- PROPOSED SYSTEM}

The proposed heuristic and exact algorithms for the periodic and non-periodic train timetabling problem on a corridor to maximize the sum of the profits of the scheduled trains. The dispatched trains from left to right and also dispatched trains from right to left form two independent sub networks of the TSP. we proposed a system that will gather all the information a human performed. In this system we will apply good clustering that cluster all relevant information of the user. We proposed auction-based scheduling algorithm, can be used efficiently in a non-cooperative situation to realize proportional fairness. We proposed auctionbased scheduling algorithm, which combines the merits of the VCG auction and the greedy MC PF algorithm, to ensure that wireless users truthfully declare their resource requirements even though the users are inherently selfish. In the proposed algorithm, it is supposed that the trains play the role of cities in the TSP. In order to minimize the total travelling time a, branch-and-bound procedure is proposed. Using a similar model to proposed a mixed integer linear model with varying departure and travelling times. The proposed model is then solved by a heuristic approach. The strategies proposed to prevent the deadlock problem in railway system are reviewed. The author concluded that the proposed models are based on the game board philosophy. 


\section{IV- MODULES OF PROPOSED PROJECT}

\section{Login Module}

The login module authenticates both the user and the admin. Once the authentication is done, the user and admin can perform their individual activities.

\section{A. Admin Module}

Admin module allows system administrator to set up backend of the system and perform basic system configuration, mainly definition predefined dropdown field, definition of classes time schedule etc. all the new packages are to be defined here like various transition and their to system times of arrival and departure. Admin can also set up is user management which allows users to be set up with definable access level/role, access to single or multiple branches. Admin can also set up overall system security setting such as required password strength, inactive session time out, inactive account lock out, password reset period, etc. only admin can access the admin module admin can enter the train details as like train name train departure train arrival time and route etc. no one can access to the admin module accept admin.

\section{B. User Module}

User can view information of train timetable user module is the module which is created for user only. User modules is also known as graphical user interface (GUI) user module is made for the easy access to user. User and admin both can access to the user module the user can only use the user module but the admin can do the changes in that user module. The start of the program the user is allowed to select a branch line for simulation and planning of the timetable. Then the user can view a table as time schedule etc. all the new packages are to be defined here like various transition and their to system times of arrival and departure. security setting such as required password strength, inactive session time out, inactive account lock out, password reset period, etc. only admin can access the admin module admin can enter the train details as like train name train departure train arrival time and route etc. no one can access to the admin module accept admin. shown. Adding a new train, changing the properties of a train such as a speed or deleting a train can be done here. When a train is added to the table it is assumed that the train is stopped at every station.
The user can change the number the number of stops for particular train using a separate window as given. The user can change the attributes of a particular train by editing it on the dialog box that appears after clicking on the train number. Users would like to see the trains run as a real-time process according to the timetable simulated by the computer. This implementation is illustrated in the window as show.

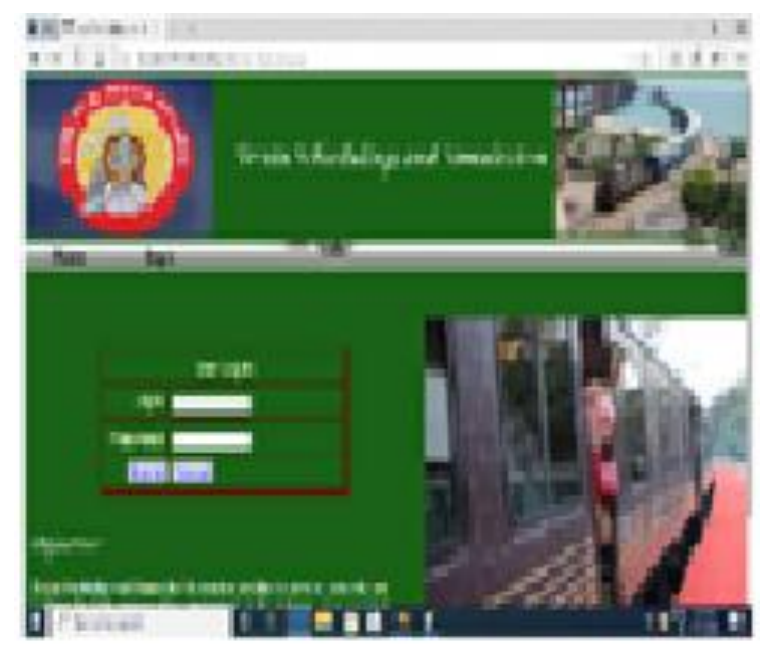

\section{User action}

Enter re-schedule (train ID, start and stop place and line, as well as lining for intermediate points).

Request to enter schedule info.

\section{System action}

Show the schedule screen.

Check that the schedule does not conflict with other existing schedule; display entered schedule for conformation.

\section{Exception}

Schedule conflict with another schedule train or maintenance task.

operator must decide whether to change the schedule or alternatively to reschedule also the other train/even. 


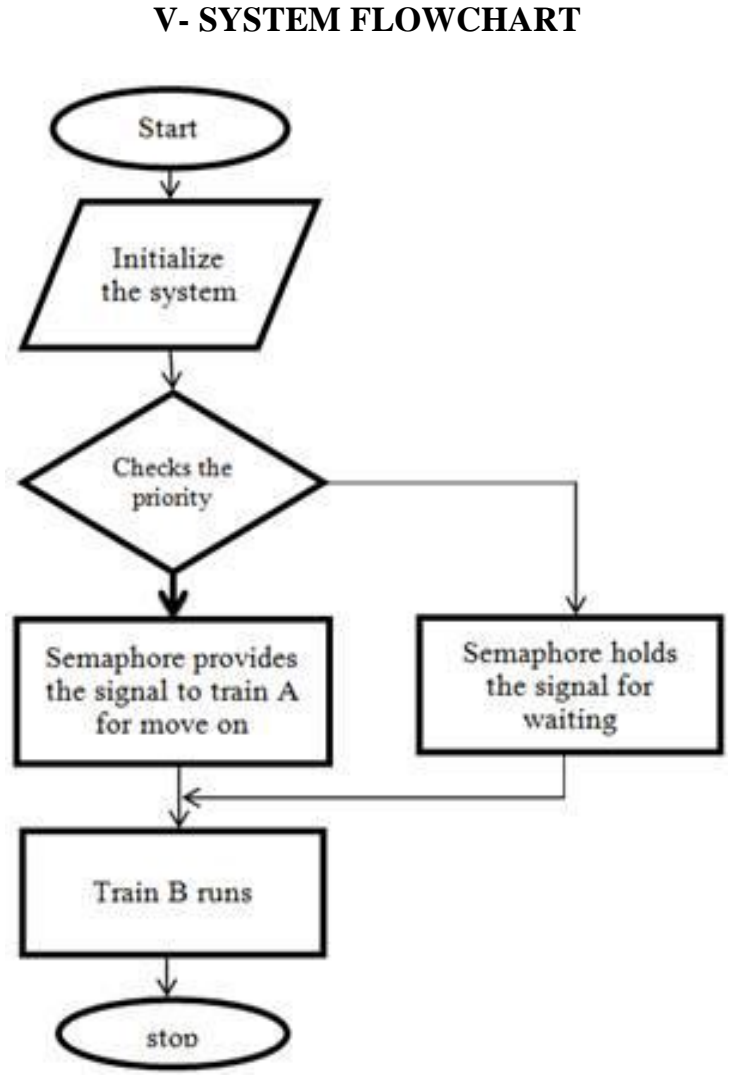

\section{VI- METHODOLOGY}

Using this simulation program it is possible to plan and optimize train schedule on single-track rail lines with trains runs within short times period. This model can be used for any single-tracks line where some station do not have siding-track and when different speed limits between station are given. For each train, the maximum speed, the number of stops on the line and the stopping time at each station can be specified.

After each run of the simulation, the results can be displayed instantly. These include the time-distance graph, the network with train run, dialog boxes with information about selected trains. Here it is also possible to change origin station, departure time, destination, and add or delete a stop etc.

There will be consist of two stages. In first stage, a simulation is used to produce a timetable without changing the predefined departure times of trains. In this process the train crossing and overtaking positions are determined so as to minimize the overall delay of trains. The simulation process is based on a very simple model. First, the trains are arranged according to the departure times and then each train is move by step by steps from the origin station to the destination with respect to a timer. It is resolved according to the dynamic priority of each train.

In second stage, scheduling is the infrastructure in the problem has a line structure inspired by a real railway line, and has a planned initial timetable with arrival and departure times of trains only at two end stations.

\section{VII- OBJECTIVE}

The objective of this paper is to develop a methodology to facilitate scheduling of trains and simulation. The paper describes the simulation model design to optimize train schedules on single-track rail lines. The multi- objective model for optimal allocation of passenger train services on a high-speed rail line has been developed by Chang, Yeh and Shen (2000). The objective was to maximize sum of the profits of the scheduled trains.

\section{VIII- IMPLEMENTATION}

This paper is implementation of the program developed to solve the train-scheduling problem is discussed in this section, starting from the input and output model, to describe the data structures used internally, the resource manager used to ensure coherent resource management, the algorithm used to produce an individual of the initial population, that is, a solution generated with a certain amount of randomness, to the techniques employed in genetic optimization. Scheduling algorithm can be used efficiently in noncooperative situation to realize proportional fairness. The rail network analysis has been implemented through networks optimization methods, including networks and simulation in particular event base simulation. The mathematical model of the problem is an integer optimization problem with side constraints, and it is difficult or impossible to solve exactly in a reasonable amount of time. In order to solve this NPhard problem, a heuristic approach was implemented that makes use genetic algorithms to find a good solution. Timetable optimization through the implementation of Opentrack has been presented by Nash and Huerlimann (2004).

\section{IX-DEVELOPMENT TOOL}

System we will be developed using Front End: html and CSS, Back End: SQL SERVER. 


\section{X- RESULT}

This result can also be generalized for some basic version of Train Scheduling using Simulation, which even have the potential to speed-up the algorithm considerably. However, the computation time of our approach increases exponentially in the number of blocks. Hence, we have implemented the project simulation model is used to produce a timetable without changing the predefined departure time of trains. This solution should satisfy the criteria for each train defined by the user i.e. the maximum delay at each station, maximum travel-time allowed.

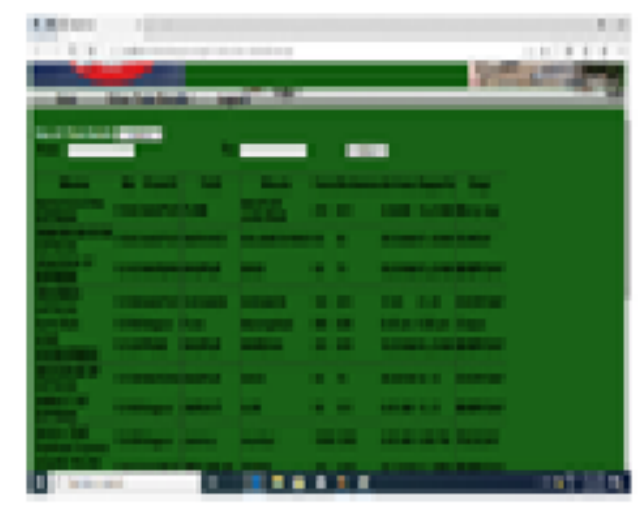

\section{XI- CONCLUSION}

In this paper we studied a basic version of the Train Scheduling using Simulation, which can be considered a special case of job shop scheduling with two counter routes and no pre-emption. Furthermore, we showed that for any fixed number of blocks (STTS) can be solved in pseudo-polynomial time. We found that, although the job shop scheduling problem with two counter routes, no pre-emption, three machines and equal number of jobs from both sides is strongly NPhard. The design concept of a train movement class is described with its properties and methods. A feasible timetable generator simulation model for the train scheduling (timetabling) problem is explained. The simulation model was developed to cope with the disturbances, therefore stochastic events were allowed in the simulation model. Therefore, the simulation framework can also be used for the train rescheduling problem if it can be feed by the real time data. A simulation run using existing train schedule that consist of 14 trains in one direction up to two destinations was executed. The resulting output will be given above table. It can be seen that departures times of some trains have been deviated so as to minimise the total travel time. Therefore, the simulation framework can also be used for the train rescheduling problem if it can be faced by the real time data. Although simulation has been used in the literature, none of them include a comprehensive framework.

\section{ACKNOWLEDGMENTS}

I would like to express my sincere gratitude to the Thailand Research Fund through the Royal Golden Jubilee Ph.D. Program for supporting this research to Chaiyut Sumpavakup. This study is supported by The Scientific and Technological Research Council of Turkey (TUBITAK).

\section{REFERENCES}

1. X Li, L Lu, P Zheng, Z Huang "Timetable Coordination of the First Trains for Subway Network" in 2019.

2. "Rail Operator Training Simulators" faac.com. FAAC. Retrieved 2019-10-04.

3. Allman, W. (2014). A network-simulation approach to the railroad freight train scheduling.

4. Zhou, Y. and Mi, C. (2013). Modelling and simulation of train movements under scheduling and control for a fixed-block railway network using cellular automata. SIMULATION, 89(6), pp.771-783.

5. Rahman, S.A.A.: Freight train scheduling on a single line network. Ph.D. thesis. The University of New South Wales (2013).

6. K. Kim, "Optimal train control on various track alignments considering speed and schedule adherence constraints", PhD Thesis, USA, January (2010).

7. Castillo, E, Gallego, I, Urena, J.M., Coronado, J.M.: Timetabling optimization of a single railway track line with sensitivity analysis. TOP 17(2), 256-287 (2009).

8. S. Q. Liu, E. Kozan, Scheduling trains as a blocking parallel-machine job shop scheduling problem, computers and Operations Research, 36 (2009) 2840 2852.

9. Babushkin, A., A., Below, I.: scheduling for the problem with oppositely directed routes. Kiber-netika 7, 130-135 (1974).

10. https://faac.com

11. https://opentrack.ch

12. https://ieeexplore.ieee.org

13. A. I. Mees, Railway scheduling by neywork optimization, Mathematical Computer Modelling, 15 (1991) 33-42.

14. Garcia and Gutierrez, 2003 "A train simulation model for strategic planning in rail Freight transport system" ITE Journel, 73(9) (2003), pp. 32-40. 
15. Cordeau et al., 1998 Cordeau J.F., Paolo T., Daniele V "A survey of optimization models for train routing

16. and scheduling" transportation science, 32(4) (1998), pp. 380-404.

17. I. Amit and D. Goldfarb, The timetable problem for railways, Developments in Operations Research 2 (1971), 379-387.

18. Sahin I., 1997, Railway traffic control and train scheduling based on inter-train conflict management, Transportation Research B vol 33, pp 511-534.

19. K. Kim, "Optimal train control on various track alignments considering speed and schedule adherence constraints", PhD Thesis, USA, January (2010).

20. D. Hurlimann, Open Track Betriebssimulation von Eisenbahnnetzen, Zurich, Switzerland, 1.6 edition, 2010.

21. D. Hurlimann, Open Track Betriebssimulation von Eisenbahnnetzen, Zurich, Switzerland, 1.6 edition, 2010.

22. Y. Cui, "simulation-Based Hybrid Model for a PartiallyAutomatic Dispatching of Railway Operation", Nordersted: Book on Demand GmbH 2010.

23. Castillo, E, Gallego, I, Urena, J.M., Coronado, J.M.: Timetabling optimization of a single railway track line with sensitivity analysis. TOP 17(2), 256-287 (2009).

24. E.R. Petersen and A. J. Taylor, "Line Block prevention in rail line dispatch and simulation models", Information System and Operation Research, vol. 21, no. 1, pp. 46-51, 1983.

25. Zhou, y. and Mi, C. (2013). Modelling and simulation of train movements under scheduling and control for a fixed-block railway network using cellular automata. SIMULATION, 89(6), PP. 771-783.

26. $S$ Yang in 2018 deals with "Energy-efficient timetable and speed profile optimization with multi-phase speed limits" focused on Energy consumption and associated costs, energy-efficient timetable and speed profile optimization for metro system have attracted much attention from both academic and industry communities. This paper develops an integrating timetable.

27. MA Murtaza, SBL Garg, in train simulation 1989, "Brake modelling in train simulation". This paper deals with brake modelling as used in train simulation study.

28. Landex and Nielsen (2013), earlier train scheduling was done by the humen experts but now with the introduction of IT and new technological tools, it is done with the help of such technological algorithms. The system keeps a check on the synchronized railway tracks and help to run the railway with the help of algorithm. The system allows scheduling of three lines of railway, i.e. it can manage the running of three railway, at a time.

29. According to Li etal. (2013), scheduling of trains helps to reducing the operation time as earlier most of the man power gets wasted in such processes but now it is done with ease. This helps organization to enhance the performance.

30. technology make work force to focus on their assigned duties rather than such scheduling processes. The complete process of scheduling the train is dependent on algorithm were the change of track, running of trains and related activities are managed with the help of algorithm.

31. Sahin I., 1997, Railway traffic control and train scheduling based on inter-train conflict management, Transportation Research B vol 33, pp 511-534.

32. J. Pachal, Deadlock avoidance in railroad operations simulations, Transportation Research Board $90^{\text {th }}$ Annual Meeting, Paper No:11-0175, Washington DC, USA, 2011.

33. U. Geske, Railway scheduling with declarative constraint programming, Lecture Notes in Artificial Intelligence, 4369 (2006) 117-134.

34. A I. Mees, Railway scheduling by network optimization, Mathematical Computer Modelling, 15 (1991) 33-42.

35. C. Liebchen, The first optimized railway timetable in practice, Transportation Science, 42 (4) (2008) 420-435.

36. B. Szpigel, Optimal train scheduling on a single track railway, Operational Research 72 (1996) 455-464.

37. U. Brannlund, P. O. Lindberg, A, Nou, J.E. Nilson, 1998, Railway timetabling using lagrangian relaxation, Transportation Science, 32 (4) (1998) 358-369.

38. Higgins, A., Kozan, E., Ferreira, L.: Optimal scheduling of trains on a single line track. Transportation Research Part B: Methodological 30 (2), 147-161 (1996).

39. D. Jovanovic, P. T. Harker, Tactical scheduling of rail operation: The SCAN I system, Transportation Science, 25 (1991) 46-64.

40. Y. Lee, C. Y. Chen, A heuristic for the train pathing and timetabling problem, Transportation Research Part B, 43 (2007) 320-341.

41. S. Q. Liu, E. Kozan, Scheduling trains as a Blocking parallel machine job shop scheduling problem, Computer and Operational Research, 36 (2009) 2840 2852. 


\section{IMPLEMENTATION OF SNAPSHOT}
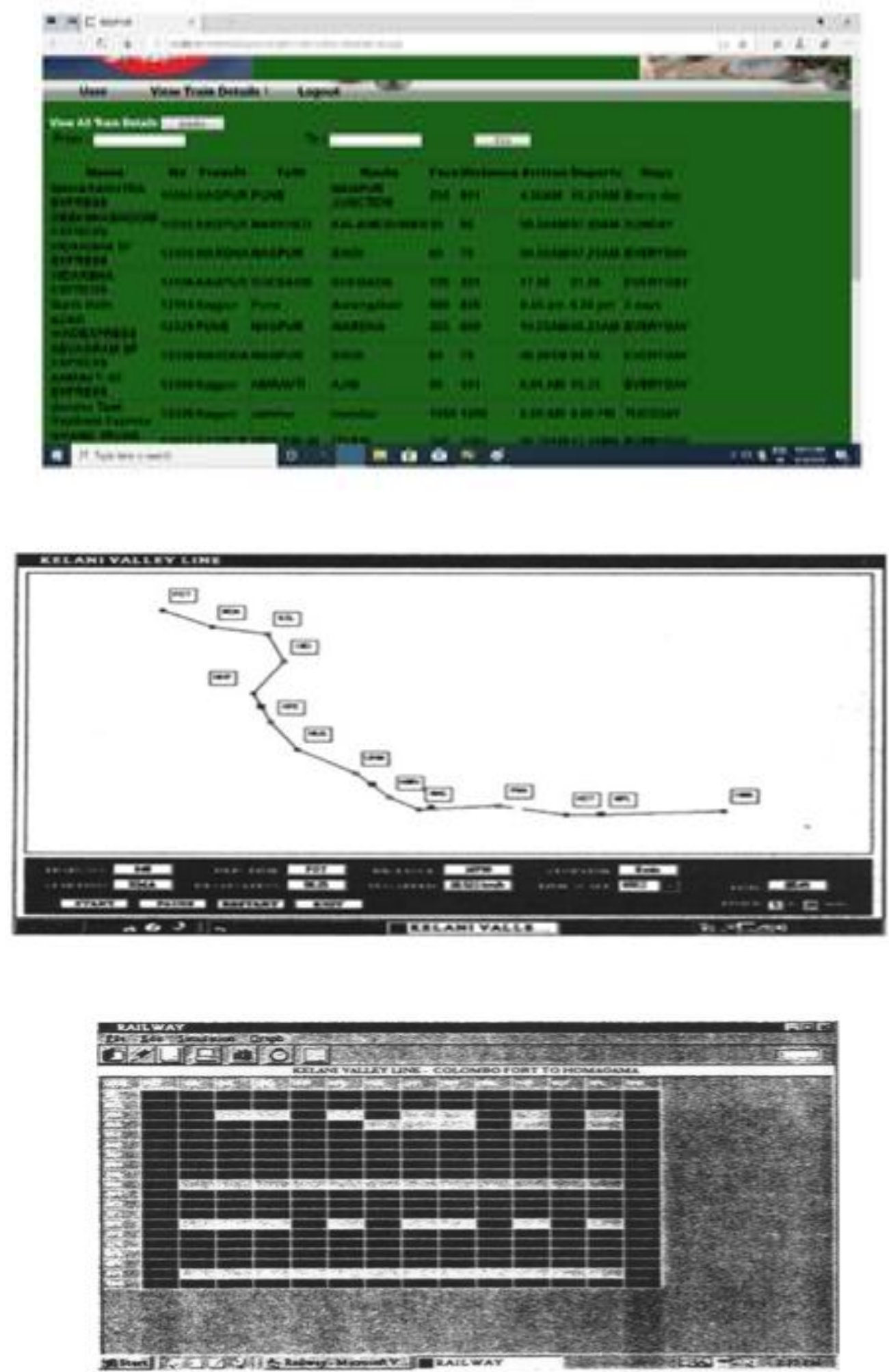

Fig. 4. Train Stop Seheduling Interface 

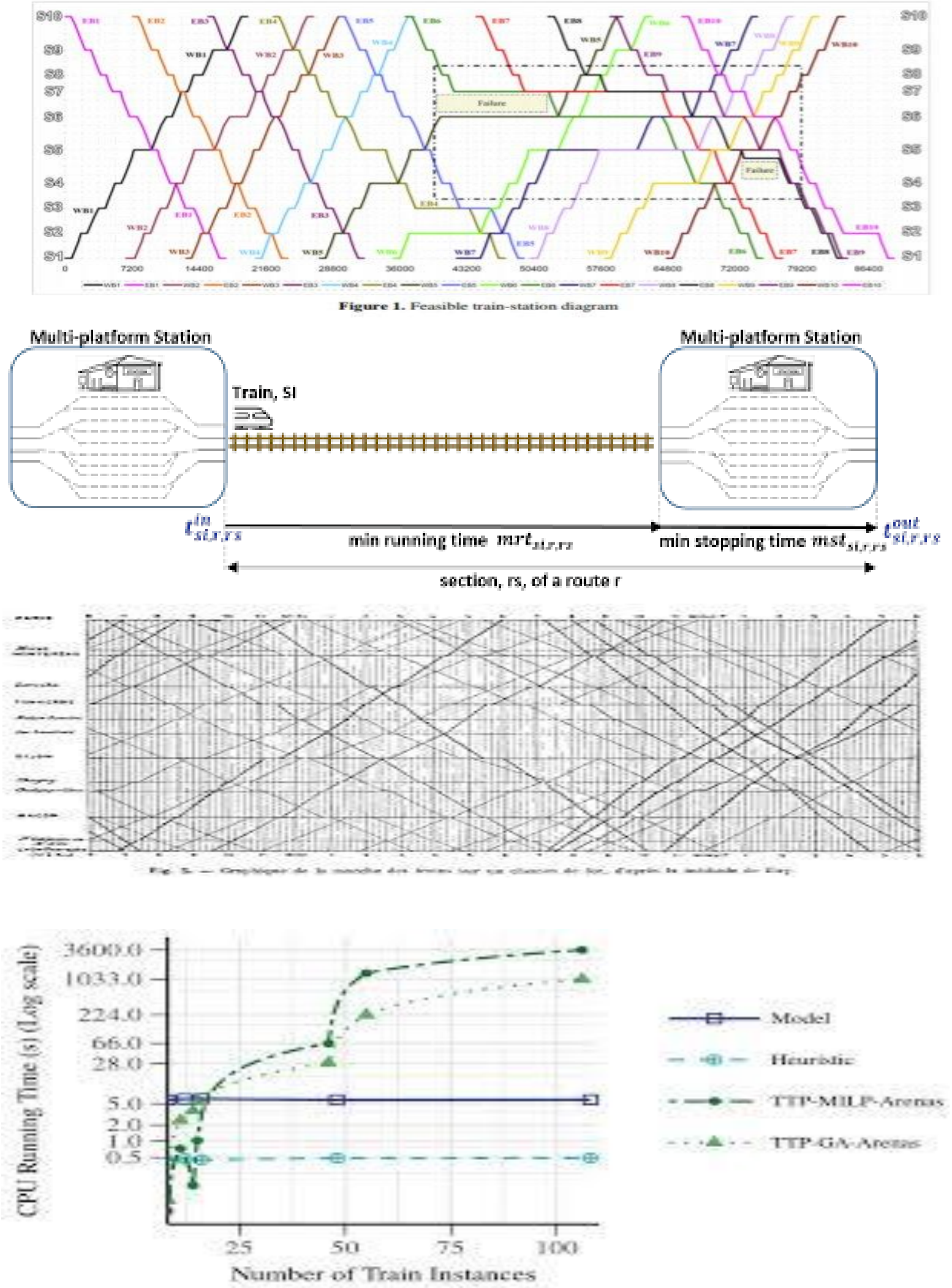

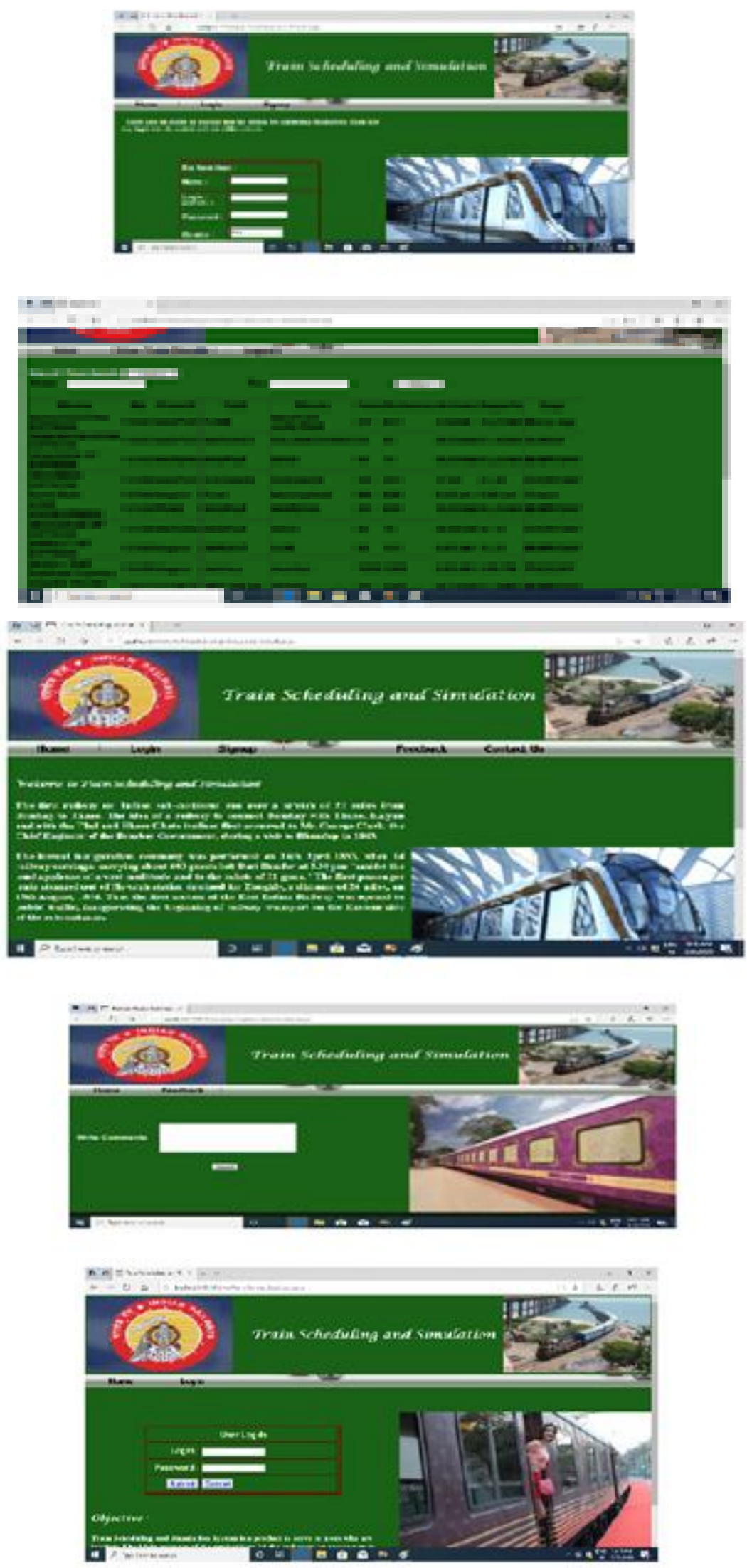